CARNETS OE Carnets de géographes

GÉOGRAPHES.

\title{
Les terrains de jeu vidéo comme terrain de recherche
}

Samuel Rufat et Hovig Ter Minassian

\section{(2) OpenEdition}

1 Journals

Édition électronique

URL : http://journals.openedition.org/cdg/2766

DOI : $10.4000 /$ cdg. 2766

ISSN : 2107-7266

Éditeur

UMR 245 - CESSMA

Référence électronique

Samuel Rufat et Hovig Ter Minassian, "Les terrains de jeu vidéo comme terrain de recherche »,

Carnets de géographes [En ligne], 2 | 2011, mis en ligne le 02 mars 2011, consulté le 07 mai 2019. URL http://journals.openedition.org/cdg/2766; DOI : 10.4000/cdg.2766

La revue Carnets de géographes est mise à disposition selon les termes de la Licence Creative Commons Attribution - Pas d'Utilisation Commerciale - Pas de Modification 4.0 International. 


\title{
Les terrains de jeu vidéo comme terrain de recherche
}

\author{
SAMUEL RUFAT \\ Maître de conférences Université de Cergy-Pontoise \\ Samuel.Rufat@ens-lyon.fr
}

HOVIG TER MINASSIAN

Maître de conférences Université François-Rabelais de Tours hterminassian@gmail.com

Lorsque les géographes, plus nombreux qu'on le pense, parlent de jeux vidéo, ils se défaussent souvent de leur pratique sur leurs descendants ou leurs proches. Rares sont les téméraires qui osent appuyer leurs remarques et leurs interrogations sur des analyses concrètes. Les jeux vidéo sont un objet en cours d'appropriation par les sciences sociales (Rufat, Ter Minassian, 2011a). Comme les jeux au début du siècle précédent, ils souffrent d'un problème de légitimité : le doute continue de peser sur le plaisir du jeu. Jouer aux jeux vidéo serait loin d'être une activité sérieuse, malgré le marketing agressif du géant japonais Nintendo ${ }^{1}$. Les jeux vidéo constituent un objet culturel porteur de nombreux discours normatifs, souvent très polarisés et la facilité d'accès au jeu vidéo - profusion des discours, disponibilité médiatique et marchande - ne pousse paradoxalement pas au débat.

Les géographes ont admis depuis longtemps qu'il était possible de faire «mentir» des cartes ou des statistiques (Monmonier, 1993), et qu'il était important de prendre en compte la manière dont les images (télévisuelles, publicitaires...) véhiculent des représentations spatiales (Gumuchian, 1991 ; Rosemberg, 2000). Alors pourquoi renoncer aux jeux vidéo ? Parce qu'il faut feindre que les jeux vidéo n'ont aucun rapport avec les pratiques et les idéologies spatiales, le marketing territorial, l'économie mondialisée et la globalisation ? Au contraire, il nous semble indispensable de donner un regard critique et des clés d'analyse des jeux vidéo aux lycéens et aux étudiants, parce qu'ils font partie du quotidien d'une majorité d'entre eux et qu'ils sont de puissants vecteurs d'idéologies et de représentations spatiales (Ter Minassian \& Rufat, 2008).

Dans cette perspective, les jeux vidéo apparaissent comme un terrain parmi d'autres. Le statut si particulier de «mondes virtuels » qu'on leur prête induit-il des méthodologies de travail différentes ? Le chercheur, cloué sur sa chaise devant un écran fait-il «moins de terrain » en parcourant les étendues d'Hyrule ou d'Azeroth qu'en arpentant les Ramblas de Barcelone ou le Palais du Peuple de Bucarest ? Où est le terrain des jeux vidéo ?

\footnotetext{
1 On peut désormais transformer sa console de jeu en podomètre, l'utiliser pour exercer sa mémoire, «apprendre» à faire la cuisine, à peindre, ou même faire de la gymnastique des yeux à l'aide d'un écran (Gym des yeux, Nintendo 2007).
} 


\section{Travailler, c'est jouer}

L'amalgame entre «terrain de recherche » et «terrain de jeu » continue de stigmatiser tout travail sur le jeu (Grataloup, 2004 ; Nace, 2008), bien plus que tout jeu sur le travail (Craipeau, 2009). D'ailleurs, dans son ouvrage Homo Ludens, Huizinga fait un trait d'esprit face à la difficulté à définir le jeu : il montre que la recherche scientifique est elle aussi une activité ludique régie par des règles et des normes (Huizinga, 1938). Malgré les réticences, il est nécessaire d'explorer les relations entre terrain de recherche et terrain de jeu. Les jeux vidéo redoublent ce problème : ils sont un terrain que l'on qualifie aisément de « virtuel ». La tentation est donc grande, pour légitimer toute réflexion sur les jeux vidéo, de montrer qu'ils sont " plus sérieux » et " plus réels » qu'il n'y parait. Mais cela conduit à passer à côté de la spécificité des jeux vidéo, en les considérant comme des supports pédagogiques ou des outils cartographiques. Bref, pour prendre les jeux vidéo au sérieux, il faut commencer par les aborder en tant que tels. Pour cela, il est nécessaire d'évacuer toutes les questions qui font écran, comme celles de légitimité, de violence ou d'addiction. Puis il faut explorer le terrain sans a priori, c'est-à-dire y jouer.

Nous appelons jeux vidéo l'ensemble des jeux qui reposent sur un programme informatique et des interactions homme/machine au travers d'interfaces (graphiques, audio et mécaniques). Ils ont trois dimensions: ce sont des simulations spatiales (dimension multimédia), qui ne miment pas le «réel » mais suscitent le plaisir du joueur (dimension ludique) et qui nécessitent que le joueur interagisse avec l'environnement simulé (interactivité). Les jeux vidéo offrent un accès au virtuel, au sens où l'entend Pierre Lévy (1998) : non pas des univers immatériels ou mis « en ligne » sur l'Internet, mais une ouverture sur l'univers des possibles. Ainsi, dans un jeu vidéo, chaque partie actualise une fraction d'un ensemble de possibilités, et cet ensemble est de plus en plus ouvert (jeux en ligne, téléchargement de contenu, modification des règles, etc.). Le joueur joue avec l'environnement simulé, mais aussi avec les temporalités, en explorant les conséquences à long terme de certaines actions, en revenant en arrière (grâce aux sauvegardes) pour en actualiser d'autres ou améliorer sa performance. Les joueurs jouent avec l'espace et le temps. Les jeux vidéo eux-mêmes " jouent » avec les émotions suscitées chez les joueurs par cette illusion d'exploration et de manipulation à volonté (esthétique, satisfaction, peur, sadisme, etc.). Ce sont ces illusions spatiales qui interpellent les géographes. Notre univers de géographe questionne notre univers de joueur (et vice versa). Transformer ces émoustillements en interrogations, c'est faire des jeux vidéo un terrain.

Ces particularités, l'actualisation au cours des parties, l'exploration simultanée de l'espace et du temps et les émotions suscitées, sont curieusement absentes des discours sur les jeux vidéo. Pourtant, ce sont elles qui imposent un travail de terrain: pour travailler sérieusement sur les jeux vidéo, il faut y jouer. Mais plusieurs centaines d'heures de jeu n'en épuisent pas les possibilités. Alors comment faire du terrain dans des espaces virtuels?

\section{Jouer, c'est travailler}

La première difficulté, en l'absence de bibliographie, c'est de faire face à l'immense variété de jeux. On en est réduit à se fier à son expérience et à son intuition. Il faut donc commencer par jouer un certain nombre d'heures à différents types de jeux pour formuler des hypothèses, puis pour pouvoir déterminer les jeux qu'il semble aisé de faire parler pour y répondre.

Ensuite, il faut l'explorer, comme n'importe quel terrain. Les jeux récents proposent d'ailleurs des outils traditionnels (simulation d'appareil photo, bloc notes) et le support informatique se prête à la création de nouveaux outils (Duplan, 2011). Il faut prendre le temps 
d'explorer les possibilités offertes et la façon dont le programme réagit aux différents choix, des plus évidents aux plus aberrants. Il faut tester tout ce qu'il est possible de faire, comme le ferait un enfant (est-il possible d'ouvrir cette porte ? que se passe-t-il si on ferme l'hôpital ? quel est le moyen de transport le plus rapide ?). C'est une démarche radicale de recherche, elle peut s'avérer longue à défaut de fastidieuse. On peut donc s'appuyer sur l'expérience d'autres joueurs, sur les guides, les forums, les blogs qui foisonnent autour des jeux vidéo. C'est aussi l'occasion de faire connaissance avec les différentes populations, au-delà de celles qui sont mises en scène ou simulées dans le jeu, et du contexte du jeu (production, distribution, utilisations, etc.).

Cette connaissance du terrain permet alors d'affiner les hypothèses, de rapprocher certains jeux, de poser les catégories de l'analyse. Il est ainsi possible de démontrer que Civilization (Microprose \& Firaxis 1991-2010) repose sur un schéma christallerien d'organisation des villes et que seules les fonctions productives de l'espace y sont simulées (Ter Minassian, Rufat, 2008), ou que Liberty City a un plan très semblable à celui de New York (Valentin, 2007), ou encore que les jeux des séries Sim City (Maxis 1989-2007) et City Life (Monte Cristo 2006-2008) sont graphiquement proches mais reposent sur des logiques spatiales très différentes. Sim City préconise le développement urbain polycentrique alors que City Life favorise la centralité et la hiérarchisation des quartiers (Rufat, Ter Minassian, 2009). Enfin, il reste à choisir l'une des nombreuses approches des sciences sociales (dont les outils informatiques comme les simulations multi-agents) pour essayer de valider ces hypothèses. Toutefois, ces transferts méthodologiques ne sont pas évidents (Valentin, 2007 ; Coavoux, 2009 ; Berry, 2009). On peut par exemple choisir une approche quantitative, en recueillant les données de sauvegarde de grappes de joueurs, mais cela pose le problème de la propriété de ces données. On peut aussi choisir une approche modélisatrice en s'appuyant sur les mécanismes du jeu, mais le code informatique des programmes n'est pas toujours facile d'accès. On peut enfin recueillir les pratiques et les représentations des joueurs et des concepteurs au travers d'entretiens approfondis, mais cela pose le problème de la représentativité de l'échantillon ${ }^{2}$, ou par de questionnaires fermés en ligne, qui sont plus faciles d'accès mais qui génèrent de nombreux biais (Coavoux, 2009). Plus généralement, Laurent Trémel évoque trois limites méthodologiques (2007) : pratiquer le jeu dans une perspective de recherche est une activité chronophage (même si l'on pourrait dire que c'est le cas de la recherche en général), qui nécessite un accès régulier à un ordinateur toujours plus puissant (mais l'équipement informatique des établissements universitaires s'est amélioré), enfin, et surtout, faire des jeux un terrain pose le problème de l'unicité du corpus.

Toutefois, ces questions de légitimité et de consistance méthodologique ne sont pas spécifiques aux jeux vidéo : c'est aussi le cas de nombreux terrains, à commencer par ceux considérés comme "marginaux» ou «exotiques» par les universitaires. De même, l'exigence de jouer le jeu pour travailler sur les jeux vidéo est commune aux démarches participatives. Ainsi, même sur un terrain plus " urbain », la réalisation d'entretiens auprès des acteurs politiques, associatifs et des techniciens, nécessite un lien de confiance qui n'est établi qu'à partir du moment où le chercheur fait preuve de sa connaissance du terrain étudié et de sa compréhension des enjeux locaux. Mais dans le cas d'un jeu vidéo, ce "seuil de confiance » est peut-être plus élevé : la profusion de discours normatifs ou polarisés et la tendance à plaquer hâtivement des thématiques sur les jeux vidéo ont suscité la méfiance des joueurs. Vincent Berry montre dans ses travaux sur World of Warcraft (Blizzard Entertainment 2004) et Dark Age of Camelot (Mythic Entertainment 2001), deux jeux de rôle en ligne dits « massivement multijoueurs » (MMORPG en anglais) et très populaires, que les joueurs considèrent d'un très mauvais œil toute intrusion de la part d'un chercheur dont la

\footnotetext{
${ }^{2}$ La plupart des sondages sur les pratiques vidéoludiques n'interrogent pas les moins de 18 ans alors que l'on sait que les pratiques vidéoludiues sont particulièrement présentes dans cette catégorie de la population.
} 
légitimité leur paraît douteuse (Berry, 2009). La réalisation d'une enquête auprès des joueurs est parfois déterminée par les conclusions des précédentes, surtout lorsque leurs méthodes et leurs hypothèses sont discutables. Les joueurs se méfient de l'intérêt qu'on leur porte, ils soupçonnent toujours un discours de stigmatisation, une nouvelle étude sur la violence ou l'addiction. Une façon de contourner cette difficulté est de « jouer le jeu » pour témoigner non pas d'une connaissance mais d'une réelle pratique du jeu, voire de s'impliquer dans les communautés dans le cas des $\mathrm{MMORPG}^{3}$. Autrement, comme le souligne Laurent Trémel, « l'observateur risque de se retrouver face aux usages d'une communauté dont il ne maîtrisera ni la grammaire ni la langue, ce biais induisant par la suite des interprétations erronées des phénomènes observés » (Trémel, 2007, p. 98).

L'importance d'établir cette relation de confiance avec les joueurs, donc de commencer par jouer, va de pair avec la nécessité de les placer au cœur de l'analyse. Comme le souligne Denis Retaillé, le géographe ne peut détacher l'analyse d'un territoire de celle de ses acteurs (Retaillé, 2010, p. 85). Ce recentrage sur les acteurs, qu'ils soient simples passants, producteurs ou habitants, prend tout son sens dans le cas d'un jeu vidéo : un jeu vidéo n'existe que par rapport à un joueur qui en actualise une partie. Sinon, il n'est qu'une suite de codes informatiques et de potentialités. C'est pourquoi, si l'on considère les jeux vidéo comme des artefacts culturels qui véhiculent des représentations spatiales et des idéologies (Fuller, Jenkins, 1995; Frasca, 2001; Trémel, 2001; Magnet, 2006; Ter Minassian \& Rufat 2008), il n'est pas suffisant de les identifier dans le contexte d'un ou plusieurs jeux (sujets, règles, images, etc.). Il faut aussi aborder la réception de ces représentations et ces idéologies: y a-t-il acceptation passive, critique, voire détournement? Comment ces représentations s'articulent-elles avec les pratiques spatiales des joueurs? Pour qu'il y ait confrontation ou transfert des représentations spatiales, il faut qu'il y ait interaction entre le joueur, la machine et les concepteurs du jeu vidéo.

\section{La virtualité des jeux vidéo repose sur un tissu d'interactions}

Les travaux sur les jeux vidéo postulent l'existence d'une interactivité spécifique qui les distinguerait d'un jeu « classique » (jeu de cartes ou jeu de plateau), du fait de la médiation de la capacité de calcul et des interfaces graphiques du support informatique. Pourtant, d'autres types de jeux partagent des caractéristiques qui semblent propres au médium vidéoludique. Comparons un jeu vidéo de tir subjectif (first person shooter ou FPS) joué en équipe et une partie de paintball jouée dans une forêt. Le scénario, les règles et la structure du jeu sont les mêmes. C'est l'ambiance ou le choix des armes qui diffère : il n'est pas possible d'utiliser le lance-roquettes de Quake III Arena (ID Software 1999) ou le canon de Unreal Tournament (Epic Games 1999-2007) dans une forêt. D'ailleurs, de nombreux jeux vidéo sont des adaptations de sports, comme Tennis for Two (1958) ou Pro Evolution Soccer (Konami 2001-2010), ou de jeux en «carton» (solitaire, Risk, Junta, Civilisation, etc) ou en «plastique» (échecs, Warhammer, Lego Star Wars, etc). Dans les jeux vidéo issus de pratiques à engagement physique (sports, courses, combat, etc.), on avance que la capacité de démultiplication du geste est propre au medium: une simple combinaison de boutons provoque la visualisation d'actions spectaculaires ${ }^{4}$. Mais dans les jeux de rôle sur papier, un simple jet de dé a la même capacité, à la seule différence que les résultats ne s'affichent pas sur un écran.

\footnotetext{
${ }^{3}$ Vincent Berry évoque même le cas d'un entretien au cours duquel l'enquêteur a dû donner des conseils au joueur pour qu'il améliore sa manière de jouer (2009)... On imagine mal un élu d'une métropole européenne demander à un doctorant de proposer des modifications à un projet urbain en cours d'élaboration.

${ }^{4}$ Voir Killer Instinct (Rareware 1994), sur la console de salon Super Nintendo, où cette logique est la plus aboutie.
} 
Pour Jesper Juul, la spécificité de l'interactivité des jeux vidéo repose plutôt dans la gestion des règles du jeu : elles sont prises en charge par le support informatique, ce qui dispense au joueur de les connaître (2005). La nature des règles dans les jeux vidéo s'oppose donc à celle des jeux en carton. Pour G. Leconte, les règles d'un jeu non informatique sont des lois au sens politique : elles sont appliquées par des joueurs, elles définissent des actions interdites (et non impossibles) et doivent être édictées pour que le jeu puisse être joué. Les lois des jeux vidéo sont au contraire nécessaires, s'appliquent immédiatement, et se découvrent progressivement : ce sont des lois au sens de lois de la nature (Leconte, 2010).

Puisque c'est la mise en retrait du joueur dans la gestion des règles qui particularise le jeu vidéo, il faut interroger l'enjeu de cette spécificité sur la manière dont les joueurs jouent et s'approprient un jeu vidéo. Ceci conduit à interroger ce qu'implique l'acte de jouer, le vécu $\mathrm{du}$ joueur en train de pratiquer un jeu vidéo, et à recentrer sur les joueurs l'étude des représentations spatiales véhiculées par les jeux vidéo. Pour ce faire, deux approches sont possibles. La première met en avant l'expérience accumulée, la "carrière vidéoludique » du joueur, pour montrer la construction au quotidien de savoirs et de compétences (Berry, 2009) et la normalisation des pratiques sociales (Coavoux, 2010). La seconde approche postule une diversité des modalités de la réception des idéologies par les joueurs (Trémel, 2001 ; Magnet, 2006 ; Ter Minassian, Rufat, 2008), mais faute de travaux empiriques les analyses ne portent souvent que sur les représentations des concepteurs de jeu vidéo.

La géographie n'est pas démunie pour aborder ce tissu d'interactions, d'autant plus qu'il n'y a pas de jeu vidéo sans espace (Stockburger, 2006 ; Günzel, 2008 ; Nitsche 2009). Les jeux vidéo sont des systèmes spatiaux qui font interagir l'espace dans le jeu vidéo, celui qui s'affiche à l'écran, l'espace du joueur, au sein duquel ont lieu les interactions hommemachine, et l'espace autour du jeu vidéo, de sa production à sa commercialisation en passant par les forums des communautés de joueurs (Rufat, Ter Minassian, 2011). C'est donc par un système spatial que se traduit l'interaction entre le joueur et le jeu. Par exemple, un même jeu vidéo ne se pratiquera pas de la même façon selon qu'il est joué sur un écran de télévision dans le salon ou sur un téléphone mobile dans le métro. De même, la pratique d'un jeu vidéo se nourrit de discussions, d'échanges et de lectures dans les forums, dans un festival ou un cybercafé. L'interactivité entre le joueur et le jeu vidéo est donc, d'après nous, moins une question de rapport au récit ou aux règles, qu'une question de mise en relation des différentes composantes du système spatial vidéoludique.

Pour aborder ce tissu d'interactions, souvent médiées et distantes, à différentes échelles, il est indispensable de croiser les méthodologies "classiques » (analyse spatiale, enquêtes, entretiens). Il est aussi possible de pratiquer l'observation participante en incarnant un ou plusieurs personnages dans les jeux multijoueurs (Turckle, 1995). Par ailleurs, l'ethnographie du virtuel a mis en place de nouvelles méthodologies, comme le lurking, ou espionnage. Il s'agit d'analyser les échanges entre les joueurs, les textes publiés en ligne ou les discussions, sans y participer. Cette démarche renvoie toutefois à une position surplombante du chercheur, qui apparait alors comme le seul censé être capable de déchiffrer ce qui est en jeu dans ce que donnent à voir les joueurs (Hine, 2000). Comme toujours, le choix d'une méthode dépend d'abord de l'objectif de l'analyse. S'il s'agit de comprendre les pratiques et les usages des joueurs, l'entretien s'impose. En revanche, s'il s'agit de "décoder» la portée idéologique ou politique d'un jeu, il faut commencer par jouer (observation participante), puis faire un travail sur la réception (enquête, entretien, ou lurking). Après tout, les jeux vidéo sont un terrain comme un autre : il faut commencer par s'approprier le terrain, puis l'interroger et choisir une méthodologie de travail en fonction d'une problématique. 


\section{Conclusion}

Jouer le jeu des jeux vidéo, c'est d'abord les considérer comme un terrain. Un terrain de jeu, parce qu'il faut commencer par y jouer pour en saisir les spécificités, et un terrain scientifique, parce que les jeux vidéo sont des systèmes spatiaux multi-scalaires aussi complexes que stimulants. Selon Frans Mäyrä, auteur d'un manuel d'analyse des jeux vidéo (2008) :

«There needs to be a dedicated academic discipline for the study of games (...). This new discipline needs to have an active dialogue with, and be building on, existing ones, as well as having its own core identity » (Mäyra, 2005).

Ce projet, qui est au cœur des Games Studies ne nous semble pas pertinent. D'une part, il y a un risque de rendre illisible la recherche sur les jeux vidéo aux yeux des non-initiés, comme le souligne Tanya Krzywinska, ancienne présidente de la Digital Games Research Association (2006). D'autre part les réflexions sur les jeux vidéo s'inscrivent dans les mêmes démarches théoriques et méthodologiques que les autres terrains qui sont devenus plus « conventionnels ». Il est indispensable de commencer par jouer pour avoir accès au terrain des jeux vidéo, mais ce terrain n'est pas «dans » les jeux ou « dans » les machines. Ce sont les territoires produits par la conception, la distribution, la pratique et la réception de ces artefacts culturels. Il ne s'agit pas seulement d'une sous-culture d'adolescents attardés, de loisirs dématérialisés ou du dernier soubresaut de la marchandisation de notre quotidien. Il s'agit d'abord de représentations et de pratiques spatiales, de la production sous nos yeux de nouveaux territoires. L'outillage des sciences sociales permet de s'approprier ce terrain comme les autres. En retour, il s'inscrit dans le recentrage actuel sur les acteurs. Quand nos laboratoires acquerront-ils des logiciels de jeu, financeront-ils des entretiens avec les concepteurs de jeux vidéo et des enquêtes auprès des joueurs au titre des frais de déplacement sur le terrain ? Comme pour l'urbain, l'enjeu pour nous géographes, c'est de comprendre ce qu'est le virtuel.

\section{BIBLIOGRAPHIE}

BERRY V., 2009, Les cadres de l'expérience virtuelle: jouer, vivre, apprendre dans un monde numérique, thèse en sciences de l'éducation sous la direction de Gilles Brougère, Université Paris 13-Villetaneuse.

BORZAKIAN M., 2009, « Que disent les jeux vidéo à la théorie ?», séminaire du laboratoire junior Jeux vidéo : Pratiques, contenus, discours, 14 octobre 2009.

COAVOUX S., 2009, "Les usages du questionnaire », journée d'études Pratiques ethnographiques sur Internet, École Normale Supérieure, Paris.

COAVOUX S., 2010, "La carrière des joueurs de World of Warcraft », in CRAIPEAU S., GENVO S., SIMONNOT B. (éd.), Les jeux vidéo au croisement du social, de l'art et de la culture, Nancy : Presses universitaires de Nancy, pp. 43-58.

CRAIPEAU S., 2009, "Jeu vidéo et travail », séminaire du laboratoire junior Jeux vidéo : Pratiques, contenus, discours, 16 décembre 2009.

DUPLAN D., « L'observation du jeu vidéo. L'angle mort de l'envers du décor? », in RUFAT S., TER MINASSIAN H. (dir.), 2011, Les jeux vidéo comme objet de recherche, Paris : Questions théoriques, pp. 29-45.

FRASCA G., 2001, Videogames of The Oppressed. Videogames As A Means For Critical Thinking And Debate, Master thesis, Georgia Insitute of Technology. 
FRASCA G., 2003, «Simulation versus narrative. Introduction to ludulogy », in WOLF M., PERRON B. (ed.), The Video Game Theory Reader, New York : Routledge, pp. 221-235.

FULLER M., JENKINS H., 1995, « Nintendo® and New World Travel Writing: A Dialogue », in JONES S. G., Cybersociety: Computer-Mediated Communication and Community, Thousand Oaks: Sage Publications, pp. 57-72.

GRATALOUP C., 2004, « Quatre moments, trois démarches, deux dimensions, une logique : Constantinople au collège », EspacesTemps.net, http://espacestemps.net/document620.html.

GUMUCHIAN H., 1991, Représentations et aménagement du territoire, Paris : Anthropos.

GÜNZEL S., 2008, «The spatial turn in computer game studies », 2nd Vienna Games

Conference Future and Reality of Gaming 2008,

https://fedora.phaidra.univie.ac.at/fedora/get/o:1741/bdef:Container/get/Guenzel The Spatial Turn in Computer_Game_Studies.pdf.

HINE C., 2000, Virtual Ethnography, Londres : Sage.

HUIZINGA J., 1938, Homo Ludens, trad. Cécile Seresia, 1988, Paris : Gallimard.

JENKINS H., 2004, " Game Design as Narrative Architecture », in WARDRIP-FRUIN N., HARRIGAN P. (ed.), First Person. New Media as Story, Performance, and Game, Cambridge MA : The MIT Press, pp. 118-130.

JUUL J., 2005, Half-Real: video games between real rules and fictional worlds, Cambridge MA : The MIT Press.

KRZYWINSKA T., 2006, "The Pleasures and Dangers of the Game. Up close and personal », Games and Culture, ${ }^{\circ} 1$ (1), pp. 119-122.

LECONTE G., 2010, « interactivité et virtualité : deux dogmes du vidéoludisme », séminaire du laboratoire junior Jeux vidéo : Pratiques, contenus, discours, 20 septembre 2010.

LÉVY P., 1998, Qu'est ce que le virtuel ?, Paris : La Découverte.

MAGNET S., 2006, « Playing at Colonization: interpreting imaginary landscapes in the video game Tropico », Journal of Communication Inquiry, ${ }^{\circ} 30$, pp. 142-162.

MÄYRÄ F., 2008, An introduction to Game Studies, Sage Publications.

MÄYRÄ F., « The quiet Revolution: three Theses for the Future of Game Studies (Hard Core Columns 4) », Digra.org, http://www.digra.org/hardcore/hc4.

MONMONIER M., 1993, Comment faire mentir les cartes, Paris : Flammarion.

NACE C., 2008, «Une pratique en classe: les simulations géographiques, jouer aujourd'hui pour être acteur demain », L'Information Géographique, vol. 72, n 3, pp. 94-98.

NITSCHE M., 2009, Video Game Spaces. Image, Play, and Structure in 3D Worlds, Cambridge MA : The MIT Press.

RETAILLÉ D., 2010, «Au terrain, un apprentissage », L'information géographique, vol. 74, $\mathrm{n}^{\circ} 1, \mathrm{pp} .84-96$.

ROSEMBERG M., Le marketing urbain en question. Production d'espace et de discours dans quatre projets de villes, Paris : Anthropos, 2000.

RUFAT S., TER MINASSIAN H., 2009, «Video Games and Emergence: new tools or new Tricks ? », colloque international Emergence in Geographical Space : Concepts, Methods and Models, Paris, 23-25 novembre 2009 (S4 European Research Group).

STOCKBURGER A., 2006, The rendered arena. Modalities of space in video and computer games, $\mathrm{Ph}$. D thesis, University of the Arts, London.

RUFAT S., TER MINASSIAN H. (dir.), 2011a, Les jeux vidéo comme objet de recherche, Paris : Questions théoriques.

RUFAT S., TER MINASSIAN H., 2011b, «Espaces et jeux vidéo », in RUFAT S., TER MINASSIAN H., (dir), Les jeux vidéo comme objet de recherche, Paris : L/P, Questions théoriques, pp. 66-87.

TER MINASSIAN H., RUFAT S., 2008, «Et si les jeux vidéo servaient à comprendre la géographie ?», Cybergeo, http://cybergeo.revues.org/17502. 
TRÉMEL L., 2001, Jeux de rôles, jeux vidéo, multimédia. Les faiseurs de mondes, Paris : PUF, 2001.

TREMEL L., 2007, « Jeux, éducation et socialisation politique : contribution au rappel de la permanence d'un processus », Géographie, Economie, Société, volume 9 n¹, pp. 83-99.

TURKLE S., 1995, Life on the screen: identity in the age of the Internet, New-York: Touchstone.

VALENTIN J., 2007, Les Espaces vidéo ludiques, vers une nouvelle approche du cyberespace, Mémoire de master 2 recherche de géographie, sous la direction de Henri Bakis, Université de Montpellier III. 